\title{
Study of an algorithm regarding production scheduling for injection mold manufacturing
}

\author{
Bogdan Pralea * and Gheorghe Nagît, \\ Gheorghe Asachi Technical University of Iasi, Department of Machine Manufacturing Technology, \\ Faculty of Machine Manufacturing and Industrial Management, Romania
}

\begin{abstract}
The objective of this study is to establish an algorithm for estimating the time necessary to manufacture an injection mold. The idea is based on the fact that one of the most crucial activities for a successful business is to correctly define the time and also cost of the product's machining hours. It will sum up various variable based on gathered data. The variable will be focused around the quantity of material manufactured during a certain period of time. This algorithm will empower the designer to give a more precise evaluation of hours and cost in shorter period of time based on the mold 3D model.
\end{abstract}

\section{Introduction}

Being part of a global economic competition, the companies need to improve and grow constantly and also they should be able to develop new capacities that answer the crucial factors of success of the markets of which they belong. The industries that have a foundation based on the injection mold manufacturing are defined as a project driven industries. Following this statement we can assume that this specific industry needs to answer the individual process characteristics. One of the biggest risks in project management is a poor estimation of the project cost and also the demands. Inside the mold manufacturing industry it is crucial to minimize risks in the early stages of the project evaluation $[1,2]$. This represents a preliminary stage where more resources are estimated. One of these important estimations revolves around the value of total manufacturing hours. It should be underlined the fact that the estimation process, is a commonly human activity and its correctness is given by the level of knowledge of the user. This in particular defines a subjective action, which can lead to an underestimation of the project resources, when the user is overconfident, or to an overvaluation of the resources when the user does not have the proper experience, which can lead to the stage when some aspect of the project could not be covered [3].

Therefore the production planning represents the main engine of competitiveness. Starting with cost reduction through the usage of resources at their maximum potential. Another fact that is presents in many companies which produce molds is that the orders from the clients are received periodically and the production capacity is not sufficient. Starting from this it is necessary to make an analysis of the components, parts that are going

\footnotetext{
*Corresponding author: bpralea@gmail.com
} 
to be produced during the next period of time. In day to day practice the production planning creations depends on the specific knowledge of the experts. Based on some key values they determine the custom made planning for each process in part.

A new approach in production planning, based on the literature, which can be defined as an active decision making tool is founded on the idea of intelligence concepts. This particular concepts are created in such a way that they process the gathered data in order to retain a better understanding of the processes and resources, both of them defined in production planning. According to some scientific articles [4] there cannot be a good definition of the intelligence concept, but there are some technologies which support this concept. Nevertheless this concept can filter information from huge data amounts and is capable to evaluate with increased flexibility. This can also serve as a warning indicator or can be turned into a vault that stores information and knowledge. In order to enable the big data transformation, this has to be collected from all types of processes which are being involved in mold manufacturing system. This data can be either generated automatically by software or inputted manually into the system [5].

In this study the authors will try to define an efficient solution for the production planning of an injection mold manufacturing industry, combining field data with existent free solution of software. This is an important concept due to the fact that companies and clients all together are changing their requirements accordingly to the market line. Moreover, different plants can have different processes with different limits. Therefore like stated above, this can impose a certain amount of flexibility to the algorithm in order to solve different type of problems. It is expected to further improve the algorithm in order to obtain more realistic plans. The developed algorithm has a specific structure suitable for a particular production system, with the possibility to add different scenarios or limits so as to cope with the specificities of each company or plant.

\section{Methodology of the experimental procedure}

As it was stated before the object of the study will be, for the authors, for this stage the developing of an algorithm which will evaluate the time necessary to complete a mold with specific processes and tools. This is an important procedure during production planning, due to the fact that base on the time value we can analyze the resources and level them so that they cannot be over allocated. The steps that define the algorithm were creating a historical database in order to have a base. In order to create the database it had to be establish which data should be collected. With this stated the authors tried to define the processes that are involve in the actual production of an injection mold.

The proposed algorithm could be capable of handling the production planning of the entire process of an injection mold, on every machine inside the manufacturing shop. For example an order enters the system from the production planning section where the operations required to produce the part are determined. After entering all types of processes inside the system the algorithm can keep track of the job status. When a scheduling is necessary the defined algorithm can generate the result in the form of Gantt chart. The phases of the individual development stages are now described.

First of all we needed do define the types of the injection mold that are being manufactured. There are three main types of molds: two-plate injection mold, three plate injection mold and off course the stack injection mold with medium and large dimensions. All these types of mold have the same component families as depicted in Figure 1. So the collected data should circle the defined type of components (Figure 2).

The families of components on which the algorithm is based on the idea that the process of the similar general parts have the same process plans about $85 \%$ of the time. For 
example, two core plates of different geometrical size and shape share basically the same operations, due to the fact that they have similar features and layout at process level.

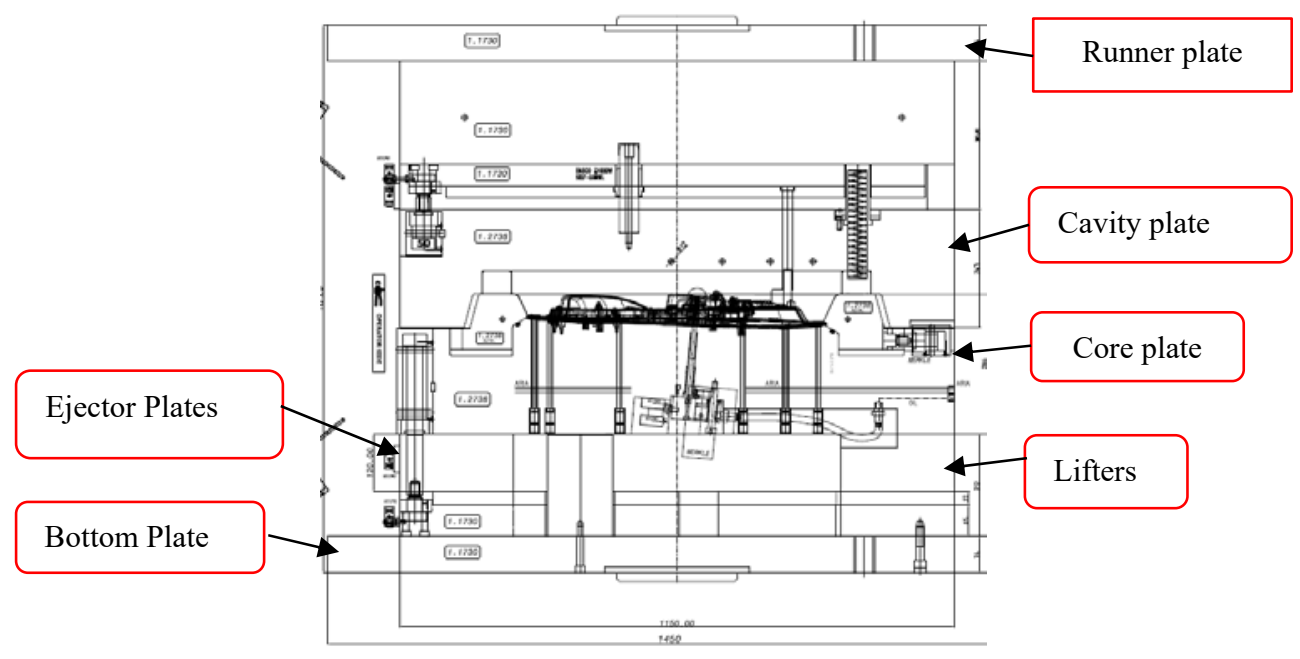

Fig. 1. The main components of an injection mold (Mold code IR47- manufactured in Trevis Grup Romania).

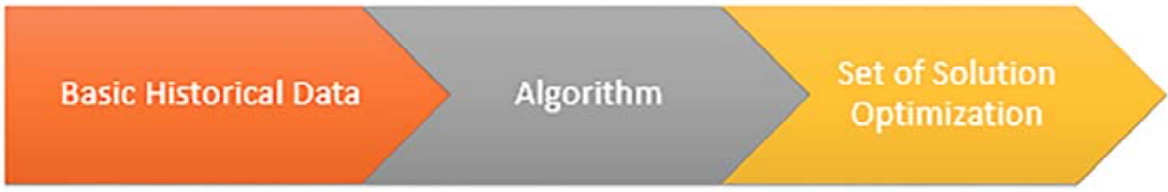

Fig. 2. The data flow of the current procedure.

The basic historical data has been collected with the help of Google Sheets software. The data has been inputted manually and afterwards double check by the user. The data collection targeted all the physical resources on the production level. The table design with the basic data is shown in Figure 3. The value that defines each resource is time. The basic data collection is carried out with the following assumptions:

- The processes of part can have any number of operations and each operation need only be processed once.

- Each process can be present a department more than once

- Each machine can only process one job a time

- All processes times include loading, set-up and unloading times

- Waiting times for cranes or forklifts are not considered as they are assumed to be always available.

In the mould manufacturing industry, paramount importance is placed on the timeliness of jobs. This is due to the competitive nature of the business where a day's difference in delivery can cost or gain the company many future projects. In comparison, potential savings from manufacturing costs such as human capacity, tooling, and raw materials are marginal to the profits generated from a single project. 


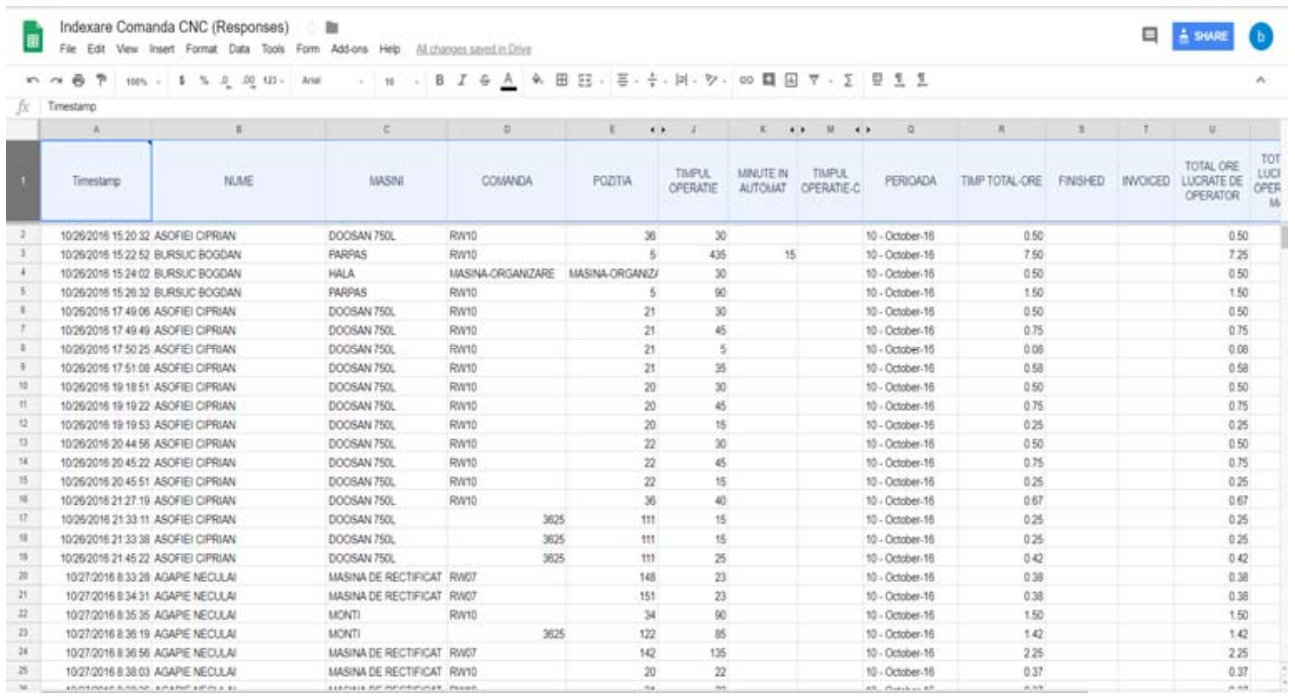

Fig. 3. The table gathers data from all physical resources, necessary for later interpretation.

It has been taken into consideration more than 80 molds of different types. Therefore the collection of data is defined on the following parameters:

- Timestamp: necessary to know on which date that particular component has been manufactured;

- The name of the operator: this involves the human touch, it is a variable that fluctuates in a matter of setup time for manufacturing a particular component. This involves different levels of expertise and knowledge;

- The machine type: each machine executes a specific operation for a specific part like milling, drilling, grinding and sawing and also it is specific to a certain component dimension;

- The mold name: this underlines the code and type of the mold;

- The component name and number;

- The engineering type of the component: each component is designed threw a CAD program and manufactured using a CAM program. Some components are analyzed threw a CAE program in order to check its integrity;

- The value of time: here it is written the amount of time in minutes necessary to complete the specific task, o a specific machine, by a specific person on a specific date.

Having defined the base procedure, it is known that each injection mold has its own bill of materials were there are defined the main geometrical value of each components. Based on the collected data, the authors determine an empirical equation which generates the future coefficients necessary for production planning. The empirical equation (1) is the key element of the algorithm:

$$
\left(W_{r}-W_{f}\right) / A_{w t}=C,
$$

Where: $W_{r}$-weight of the raw part; $W_{f}$-weight of the finish part; $A_{w t}$-actual work time; $C$ - the coefficient value.

For each family of components we applied this equation and determine an average number from more than 80 inspected molds. Afterwards we inserted the data in Table 1. 
Table 1. The coefficient Value of each family.

\begin{tabular}{|c|c|}
\hline Family name & Coefficient Value \\
\hline Top plate (Runner Plate) & 4.6 \\
\hline Bottom Plate & 3.79 \\
\hline Cavity plate & 1.59 \\
\hline Core Plate & 1.42 \\
\hline Rails & 2.67 \\
\hline Ejector plate & 4.7 \\
\hline Ejector pin Retainer plate & 2.27 \\
\hline Sliders & 1.07 \\
\hline Various parts $\geq 100 \times 100$ & 1.85 \\
\hline Various parts $\leq 100 x 100$ & 0.73 \\
\hline Parts that need heat treatment & 0.73 \\
\hline
\end{tabular}

After having determined the coefficients we started building a macro program in Microsoft Excel. The program reads the bill of materials, searches for the main dimensions in order to define the weight of the raw part, than reads the values from the bill of materials in order to insert the weight for the finished part. After it calculates the difference between the two values. Based on type of component it is put inside a category were it is multiply by the coefficient value.

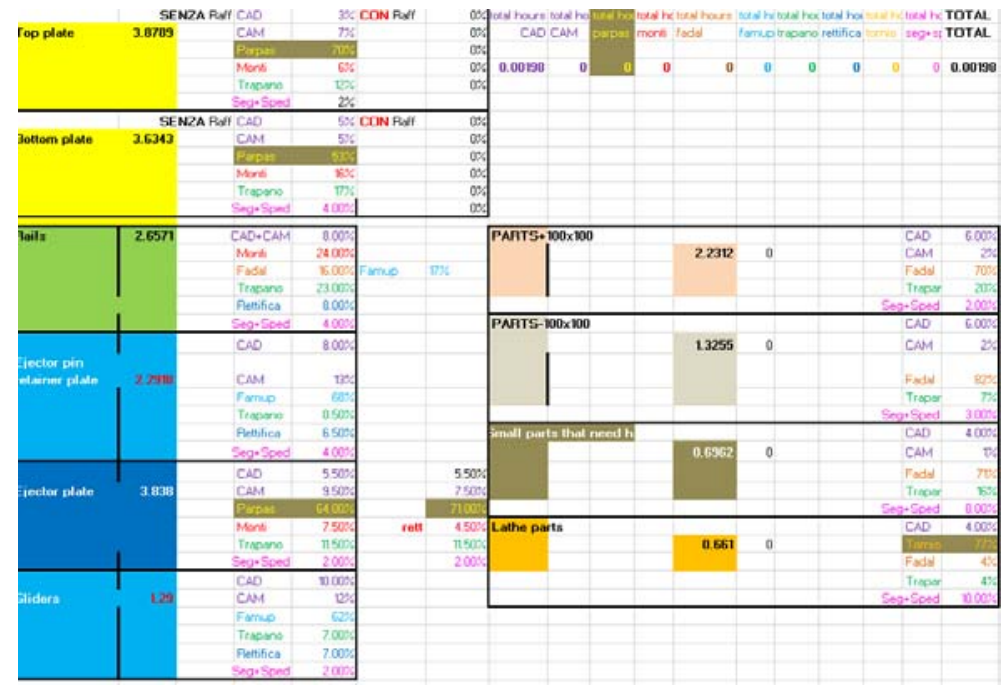

Fig. 4. The breakdown of the component's manufacturing stages.

The resulted value represents the total number of hours which are needed to complete the part. With this particular value we can also generate the necessary percentages in order to find the exact amount of time that is going to be spent at each process Figure 4. These numbers can be further inputted into a Gant Chart were we can visualize the exact time limits of our project. 


\section{Conclusions}

This study focuses on production planning, which is one of most important information process. The implementation of the approach based part-driven features gives an expert instruction on how to process basic response from the manufacturing shop in order to achieve acceptable estimation confidence. The algorithm principles were based on the classification and grouping component parts of an injection mould into families, divide those families onto machine at workshop level, create database with time and complexity for each individual family, define the specific coefficient which evaluates the quantity of material manufactured between raw and finish part. This approach is conservative. The major role in this estimation process has a very specific production environment (individual production process) with consequentially limited number of cases on one hand, and on the other hand the assumption cannot be neglected that by implementing a limited number of parameters the information is incomplete. To sum up, the authors think that this algorithm is subject to further improvements to finally represent a scheduling system for flexible operations inside a mould manufacturing shop.

\section{References}

1. M. Hoffmann, K. Kreisköther, Optimized Factory Planning and Process Chain Formation Using Virtual Production Intelligence, C.A.R.V. (2013)

2. L. Mercl, An Algorithm for Migration and Resource Planning in Cloud Technologies, Lecture Notes on Data Engineering and Communications Technologies 13 (2018)

3. Z. Jiang, Z. Fan, J. W. Sutherland, H. Zhang, X. Zhang, Development of an optimal method for remanufacturing process plan selection, Int J Adv Manuf Technol 72, 9-12 (2014)

4. F. Zhang, Y. F. Zhang, A. Y. C. Nee, Using genetic algorithms in process planning for job shop machining, IEEE Transactions on Evolutionary Computation, 4, (1997)

5. P. Y. Gan, K. S. Lee, Y. F. Zhang, C. Y. Hung, Computer aided process planning system based on branch and bound algorithm, C.A.P Eng. Conf., 7, (2000) 